\title{
Claudin-18: unexpected regulator of lung alveolar epithelial cell proliferation
}

\author{
Darrell N. Kotton \\ Center for Regenerative Medicine, Boston University and Boston Medical Center, Boston, Massachusetts, USA. The Pulmonary Center and Department of Medicine, \\ Boston University School of Medicine, Boston, Massachusetts, USA.
}

\begin{abstract}
Claudin 18 (CLDN18) is a tight junction protein that is highly expressed in the lung. While mice lacking CLDN18 exhibit the expected loss of epithelial integrity in the lung, these animals also have unexpectedly large lungs. In this issue of the $J C I$, Zhou, Flodby, and colleagues reveal that the increased lung size of $\mathrm{Cldn} 18^{-/-}$mice is the result of increased type 2 alveolar epithelial (AT2) cell proliferation. This increase in proliferation was shown to be driven by translocation of the transcriptional regulator Yes-associated protein (YAP) to the nucleus and subsequent induction of proliferative pathways. CLDN18-deficent mice also had increased frequency of lung adenocarcinomas. Together, the results of this study advance our understanding of the mechanisms that likely regulate homeostasis of the normal lung as well as promote the proliferative state of malignant cells found in lung adenocarcinomas thought to originate from AT2 cells.
\end{abstract}

\section{Loss of junction protein increases lung size}

Previous studies by Li et al. (1) and LaFemina et al. (2) have shown that mice lacking the tight junction protein claudin 18 (CLDN18), which is highly expressed in lung alveolar epithelium, have an expected defect in epithelial barrier function in the alveoli as the result of defective epithelial apical tight junctions. Unexpectedly, CLDN18-deficent animals are also characterized by lungs that are notably larger than those of WT mice. In this issue, Zhou, Flodby, and colleagues further examine the role of CLDN18 in an effort to understand why mice lacking in this protein have larger lungs (3). Their study reveals important mechanisms that regulate proliferation of the facultative progenitors of lung alveoli, type 2 alveolar epithelial (AT2) cells. The authors have identified an elegant signaling cascade whereby distal lung tissue potentially senses and responds to disruptions in cellcell contacts between AT2 cells and their neighbors by activating proliferation of the affected AT2 cells via nuclear translocation of YAP.

Like many interesting discoveries, the work by Zhou, Flodby et al. resulted from studying an unexpected phenotype in a knockout mouse model, namely the unexpectedly large lungs observed in CLDN18-deficient mice. Zhou, Flodby, and colleagues determined that proliferative indices were increased in AT2 cells of $\mathrm{Cldn} 18^{-/-}$mice, thus narrowing the search for the cellular culprits responsible for the increased organ size down to the facultative progenitors of the alveolar epithelium. Importantly, an increase in AT2 cell number, rather than an increase in cell size, was shown to explain the enlarged lungs of CLD18-deficent mice. Moreover, these changes in proliferative indices were detectable in the developing mouse lung by embryonic day 18. Additionally, Zhou, Flodby, and colleagues evaluated if the observed effects of

Related Article: p. 970

Conflict of interest: The author has declared that no conflict of interest exists.

Reference information: / Clin Invest. 2018;128(3):903-905. https://doi.org/10.1172/JCI99799.

Cldn18 deletion were detectable postnatally and were intrinsic to lung epithelial perturbations. The enlarged lung phenotype was carefully replicated in adult animals with AT2-specific, tamoxifeninducible deletion of Cldn18. Importantly, perturbation of this apical tight junction protein in AT2 cells of adult mice directly increased AT 2 cell proliferation. As AT2 cells are normally quiescent in uninjured lung tissue, understanding mechanisms that promote AT2 cells to reenter into the cell cycle are important, because proliferating AT2 cells have potential to be harnessed for lung regeneration.

\section{CLDN18 deficiency sends YAP to the nucleus}

An emerging body of literature has implicated several pathways in promoting proliferation of lung alveolar progenitors, either during development or in adults. For example, FGF, Wnt, activated KRAS, and, most recently, YAP signaling have all been reported to promote alveolar epithelial cell proliferation (4-9). However, the utility of targeting these pathways to specifically enhance lung alveolar progenitor proliferation is limited, as their initiating ligands and/or receptors tend to be broadly expressed in multiple organs during many developmental time points. Thus, it has been unclear if there might be lung epithelium-specific- sensors of injury, mechanical stress, or barrier disruption in adults that could rapidly initiate signaling through one or more of these proproliferative pathways. The findings by Zhou, Flodby, et al. are exciting and suggest that the 18.1 isoform of CLDN18, which is a relatively lung-specific tight junction protein, can act to directly initiate YAP activity in AT2 cells, thus revealing that the site of cell-cell contacts modulate lung alveolar progenitor cell cycle and function. Through a series of elegant mouse genetic models and in vitro studies to evaluate Cldn18 deletion, knockdown, or overex- 
A

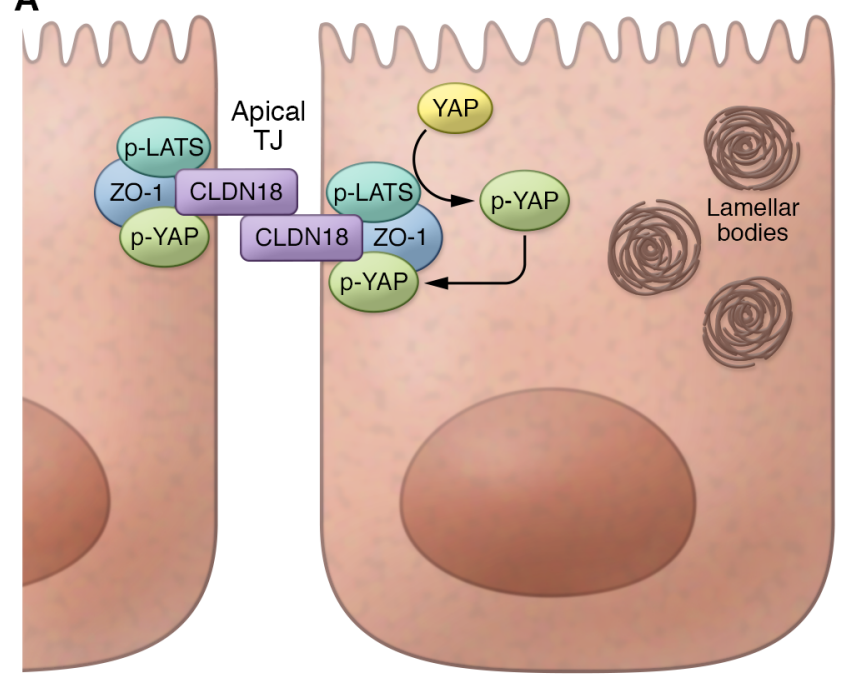

B

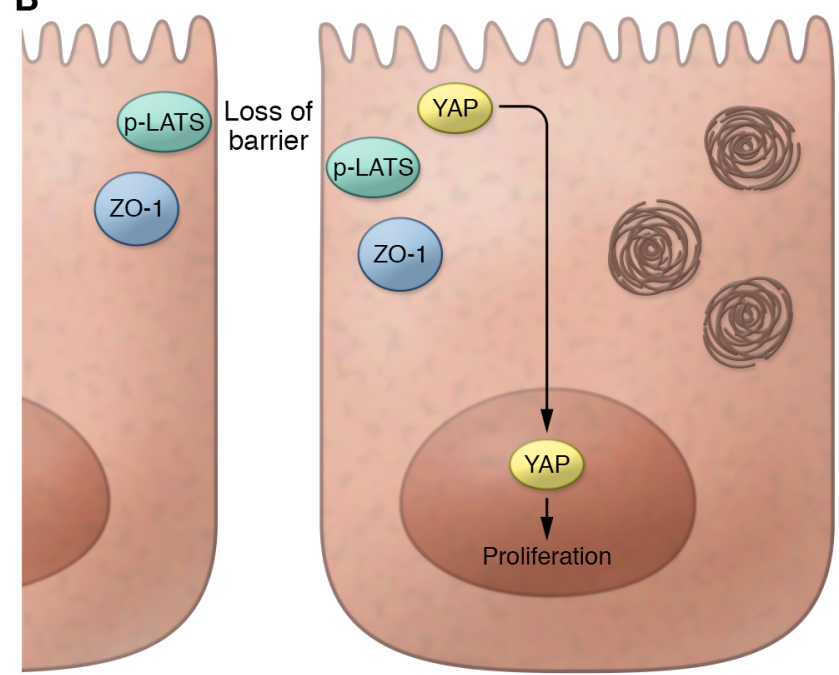

Figure 1. Schematic summarizing the effects of CLDN18 deficiency in type 2 alveolar epithelial (AT2) cells. (A) Normally CLDN18 in AT2 cells is found as part of a membrane complex in epithelial apical tight junctions, bound to the tight junction (TJ) protein ZO-1, as well bound to Hippo kinases (p-LATS), and phosphorylated YAP (p-YAP). The phosphorylation of YAP by p-LATS sequesters p-YAP protein in the cytoplasm bound to CLDN18 in the apical junction protein complex. (B) In contrast, loss of CLDN18 in AT2 cells results in loss of tight junction epithelial barrier function as well as loss of p-LATS activity, allowing dephosphorylated YAP to translocate to the nucleus where it promotes proliferation.

pression, Zhou, Flodby et al. conclude that CLDN18 in apical tight junctions of AT2 cells directly associates with ZO-1, phosphorylated YAP (p-YAP), and Hippo pathway kinases (p-LATS1/2). Therefore, loss of CLDN18 disrupts this membrane protein complex, leading to nuclear translocation of dephosphorylated YAP and induction of the AT2 cell cycle (Figure 1).

YAP has previously been shown to have a variety of important roles in lung epithelial cells. For example, YAP activation was recently shown to promote AT2 cell proliferation in mice during compensatory lung growth following pneumonectomy (9). In addition, a growing body of literature implicates YAP as a regulator of airway epithelial cell developmental patterning, differentiation, and proliferation (10-12). Analogous to the current work by Zhou, Flodby, and colleagues, Varelas and colleagues demonstrated that in airway epithelia, the apically polarized crumbs protein CRB3 promotes interactions between YAP and Hippo kinases at apical junctions, thus contributing to cytoplasmic retention of p-YAP (10). Loss of $\mathrm{Crb3}$ and consequent nuclear translocation of dephosphorylated YAP promotes emergence of basal airway progenitors while blocking their differentiation (10). In contrast, Zhou, Flodby et al. have shown that YAP-driven AT2 cell proliferation in their model is accompanied by an intact capacity to differentiate into type 1 alveolar (AT1) epithelial progeny. Taken together, the work significantly extends the recently reported observations of a role for YAP in lung epithelial cells, particularly related to AT2 cell proliferation.

\section{Clinical implications and future directions}

The discovery that loss of CLDN18 increases proliferation of alveolar progenitors capable of giving rise to both AT1 and AT2 cells has several important clinical implications and raises the intriguing possibility that pharmacological modulation of either CLDN18 or its binding partner YAP has potential to promote lung regeneration or alveolar repair after injury. The development of a therapeutic strategy to induce lung regeneration is a very high hurdle that has yet to be surmounted in clinical trials, despite the enormous burden of morbidity and mortality that results from diseases that involve the alveolar epithelium, such as acute lung injury, acute respiratory distress syndrome (ARDS), and pulmonary fibrosis. As is the case in many tissues, however, there is a fine line between appropriate progenitor cell proliferation during repair after injury and unrestrained proliferation during oncogenesis. Like several other proproliferative regulators, YAP has been implicated in many malignancies, and Zhou, Flodby, and colleagues found that in addition to activating nuclear YAP and inducing AT2 cell proliferation, reduced CLDN18 also led to the emergence of lung adenocarcinomas in aging Cldn18-null mice. Intriguingly, human lung adenocarcinomas, which are proposed to be of AT2 cell origin, exhibited this same pattern of reduced CLDN18 and increased nuclear YAP. Thus, the work by Zhou, Flodby, and colleagues nicely exemplifies both the promise and perils of augmenting YAP activity in epithelial progenitors via CLDN18 perturbations.

CLDN18 is a logical target for lung researchers to interrogate, as it is one of the claudins most highly expressed in the alveolar epithelium and its gene has binding sites for the lung epithelium-selective transcription factor, NKX2-1 $(1,2,13,14)$. It should be noted that organ enlargement in Cldn18null mice was not limited to the lung and extended to other organs, including the stomach and kidney, that are known to express CLDN18. However, the identity of the progenitor cell types responsible for and the contribution of downstream YAP-regulated mechanisms to increased organ size in other CLDN18-expressing tissues remain unclear.

Given the growing interest of the research community in understanding how lung progenitors sense injury, reenter the cell cycle, and potentially regenerate alveoli, a number of additional interesting questions are also raised by the present 
work. For example, are other pathways, such as Wnt, FGF, or KRAS signaling, proposed to promote AT2 cell proliferation, activated by reduced CLDN18? Do disrupted cell-cell contacts or reduced junctional integrity in alveoli selectively regulate YAP activity or more generally activate a diverse variety of factors that drive AT 2 cell proliferation? In addition, it will be interesting to discern the order in which cytoplasmic p-YAP- and CLDN18mediated tight junctional integrity is reestablished after epithelial cell numbers have been reconstituted. Finally, the downstream transcriptional regulators that drive AT2 cell proliferation, even if predominantly driven by YAP, remain almost entirely unstudied and likely represent the keys to promoting effective lung regeneration in the years ahead.

\section{Acknowledgments}

D.N. Kotton is supported by NIH grants

R01 HL095993, U01HL134745, and U01HL134766.
Address correspondence to: Darrell N. Kotton, 670 Albany Street, 2nd floor CReM, Boston, Massachusetts 02118, USA. Phone: 617.414.2969; Email: dkotton@bu.edu.

1. Li G, et al. Knockout mice reveal key roles for claudin 18 in alveolar barrier properties and fluid homeostasis. Am J Respir Cell Mol Biol. 2014;51(2):210-222.

2. LaFemina MJ, et al. Claudin-18 deficiency results in alveolar barrier dysfunction and impaired alveologenesis in mice. Am J Respir Cell Mol Biol. 2014;51(4):550-558.

3. Zhou B, et al. Claudin-18-mediated YAP activity regulates lung stem and progenitor cell homeostasis and tumorigenesis. JClin Invest. 2018;128(3):970-984.

4. Barkauskas CE, et al. Type 2 alveolar cells are stem cells in adult lung. J Clin Invest. 2013;123(7):3025-3036

5. Morrisey EE, Hogan BL. Preparing for the first breath: genetic and cellular mechanisms in lung development. Dev Cell. 2010;18(1):8-23.

6. Desai TJ, Brownfield DG, Krasnow MA. Alveolar progenitor and stem cells in lung development, renewal and cancer. Nature. 2014;507(7491):190-194.

7. Alanis DM, Chang DR, Akiyama H, Krasnow MA, Chen J. Two nested developmental waves demarcate a compartment boundary in the mouse lung. Nat Commun. 2014;5:3923.

8. Jacob A, et al. Differentiation of human pluripotent stem cells into functional lung alveolar epithelial cells. Cell Stem Cell. 2017;21(4):472-488.e10.

9. Liu Z, et al. MAPK-mediated YAP activation controls mechanical-tension-induced pulmonary alveolar regeneration. Cell Rep. 2016;16(7):1810-1819.

10. Szymaniak AD, Mahoney JE, Cardoso WV, Varelas X. Crumbs3-mediated polarity directs airway epithelial cell fate through the Hippo pathway effector Yap. Dev Cell. 2015;34(3):283-296.

11. Mahoney JE, Mori M, Szymaniak AD, Varelas X, Cardoso WV. The hippo pathway effector Yap controls patterning and differentiation of airway epithelial progenitors. Dev Cell. 2014;30(2):137-150.

12. Zhao R, et al. Yap tunes airway epithelial size and architecture by regulating the identity, maintenance, and self-renewal of stem cells. Dev Cell. 2014;30(2):151-165.

13. Schlingmann B, Molina SA, Koval M. Claudins: gatekeepers of lung epithelial function. Semin Cell Dev Biol. 2015;42:47-57.

14. Niimi T, et al. claudin-18, a novel downstream target gene for the T/EBP/NKX2.1 homeodomain transcription factor, encodes lung- and stomachspecific isoforms through alternative splicing. $\mathrm{Mol}$ Cell Biol. 2001;21(21):7380-7390. 